\title{
La représentation des émotions : le motif floral de Chaucer à Shakespeare
}

\section{Nathalie Fauré}

\section{(2) OpenEdition \\ 12 Journals}

Édition électronique

URL : http://journals.openedition.org/shakespeare/906

DOI : 10.4000/shakespeare.906

ISSN : 2271-6424

Éditeur

Société Française Shakespeare

Édition imprimée

Date de publication : 1 novembre 2002

Pagination : 49-69

\section{Référence électronique}

Nathalie Fauré, "La représentation des émotions : le motif floral de Chaucer à Shakespeare », Actes des congrès de la Société française Shakespeare [En ligne], 19 | 2002, mis en ligne le 01 novembre 2007, consulté le 10 décembre 2020. URL : http://journals.openedition.org/shakespeare/906 ; DOI : https:// doi.org/10.4000/shakespeare.906 


\section{S H A K E S P E A R E \\ \& L E M O Y E N - Â G E}

Société Française Shakespeare

Actes du Congrès de 2001

* * *

Textes réunis et présentés par

Patricia DORVAL

publiés sous la direction de Jean-Marie MAGUIN 


\section{LA REPRÉSENTATION DES ÉMOTIONS: \\ LE MOTIF FLORAL DE CHAUCER À S H A K E S P E A R E}

L'écriture poétique s'est lentement mise en place avec ce qui fut l'Âge d'Or de la Scolastique 'qui suivit la fin de la théologie spéculative ${ }^{2}$. Pour ce qui est de la littérature et de la poésie, les XIIIe et XIVe siècles sont marqués par la poésie des troubadours ; le poids de la spiritualité bride encore la rhétorique, saturée de métaphores mystiques et incapable de se défaire d'une écriture décorative où l'adresse à un double destinataire, Dieu et l'être aimé, est confondue.

Si une écriture de l'émotion a pu, malgré tout, durant cette période, prendre son essor, c'est grâce à l'émergence d'une instance narrative, qui, jouant sur l'écart entre le récit d'événements et le discours, est lentement devenue un «je» lyrique et aussi, par extension, un narrateur préromanesque, voire un romancier. Nous parlerons donc de métalepse énonciative ${ }^{3}$ pour désigner le passage d'un niveau narratif à l'autre.

Parler de la représentation des émotions signifie aussi que l'avènement de ce «je» lyrique, sorte de parolier ventriloque, repose sur un effet de sens qui n'est pas loin de celui produit par la figure de rhétorique appelée «synesthésie», dont nous retiendrons la définition suivante :

Synesthesia is the psychological term for experiencing two or more kinds of sensation when 
only one sense is being stimulated. In literature the term is applied to descriptions of one kind of sensation in terms of another ; color is attributed to sounds, odor to colors, sound to odors, and so on. [...] Synesthesia [...] is sometimes also called 'sense transference' or 'sense analogy' [...].

Cette définition distingue entre la figure de rhétorique et l'expérience psychologique, en l'occurrence le champ sémantique des sensations différentes éveillées par un sens unique ; la vue suggérant le goût ou le toucher, ou encore les sonorités et vice versa. Il y a là un effet de sens qui, selon nous, est au ccur de textes poétiques que nous allons citer, tous centrés sur le motif floral. Ainsi, l'analogie entre des sens différents, la vue pour l'ouïe en particulier, est un mot clef pour appréhender la nature des émotions.

Au Moyen-Âge, l'esthétique est gouvernée par l'ex-position, c'est-àdire la représentation des idées fondée sur le lien analogique qui relie l'homme à la nature. Cette relation microcosme / macrocosme est surtout d'ordre visuel, et donc la synesthésie ne peut qu'être envisagée selon une perspective restreinte. Ainsi relèverons-nous dans la poésie chaucérienne le prélude d'émotions pressenties, véhiculées par l'écriture elle-même. Le passage à l'écriture de la Renaissance a dépassé, voire embrassé la dualité homme / nature en un tout qui se voulait désormais plus harmonieux. Ce processus de métamorphose et d'enrichissement de la pensée et du langage a donné jour à ce que Bachelard qualifie «d'instant poétique», processus selon lequel le «temps travaillé» implique des transferts ou des changements sur le plan lexical ${ }^{5}$. Ainsi, le lexique usité au Moyen-Âge, en l'occurrence celui du floral, s'est peu à peu lexicalisé pendant la Renaissance et s'est surtout, comme nous le verrons plus loin, ajusté au registre humain comme «partie intégrante de son expressivité», pour reprendre la définition citée précédemment.

Notre étude s'efforcera de rappeler l'évolution du motif floral depuis la littérature gothique jusqu'au théâtre élisabéthain : nous nous pencherons sur la notion d'instant poétique relative à la nature du motif étudié, soit médiéval soit maniériste (et cela en portant une attention particulière à la courbe syntagmatique et paradigmatique du texte poétique). Les exemples étudiés vont mettre en lumière les différentes valeurs poétiques de ce motif, et rendront compte de l'émotion informulée qui émane d'un texte 
poétique et le transcende tout à la fois, démystifiant de ce fait le langage de la passion et le ramenant à ses justes valeurs humaines.

Ainsi, nous ferons d'abord le parcours du floral dans le temps, après quoi nous tenterons de percer les mystères de la «langue des oiseaux», terme emprunté aux poètes courtois.

\section{Du motif courtois au motif élisabéthain : l'évolution du motif floral anglais}

L'étude d'un motif comme celui du floral implique, chez le lecteur, non seulement d'envisager la valeur ou les valeurs poétiques du signe mais aussi la place qu'il occupe au sein du texte littéraire, c'est-à-dire sa mise en contexte. Celle-ci évolue au fil du temps et les facteurs politico-socioculturels ne sont pas à exclure. Il semblerait que ce que le floral a perdu dans son parcours poétique, en l'occurrence ses significations spirituelles et plus simplement le goût de l'allégorie, les poètes de la Renaissance en aient tiré des leçons, transformant le paysage littéraire jusqu'alors figé en un spectacle vivant et animé.

Dans le roman chevaleresque de Geoffrey Chaucer, The Knightes Tale, les figures de rhétorique font bel et bien défaut au conteur qui se cantonne à utiliser la catachrèse (ou métaphore figée) et la prosopopée appliquée au proverbe. Cette entropie lexicale qui résulte d'un parler décoratif, banalisé par les canons esthétiques de la littérature courtoise héritée du Moyen-Âge, n'émeut guère le lecteur. Celui-ci finit par perdre de vue l'instant subliminal et émotionnel que le texte cherche malgré tout à afficher, quoique par des moyens qui demeurent trop souvent archaïques. En revanche, ce que la rhétorique ne peut accomplir, la syntaxe y parvient, et cela par une simple inflexion dans le phrasé, qui comme nous le verrons, redonne vie et épaisseur au texte.

\section{1) La Fleur chez Chaucer}

\section{a) La fleur et la voix du poète}

But sooth is seyd, gon sithen many yeres, That 'feeld hath eyen, and the wode hath eres'.

That speces of thinges and progressiouns

Shullen enduren by successiouns, 
And nat eterne be, with-oute lyë :

This maistow understonde and seen at yë.

'Lo the ook, that hath so long a norisshinge

From tyme that it first biginneth springe,

And hath so long a lyf, as we may see,

Yet at the laste wasted is the tree.

'Considereth eek, how that the harde stoon

Under our feet, on which we trede and goon,

Yit wasteth it, as it lyth by the weye.

The brode river somtyme wexeth dreye.

The grete tounes see we wane and wende.

Than may ye see that al this thing hath ende.

'Of man and womman seen we wel also, that nedeth, in oon of thise termes two,

This is to seyn, in youthe or elles age,

He moot ben deed, the king as shal a page ;

(3013-30)

And certeinly a man hath most honour

To dyen in his excellence and flour,

Whan he is siker of his gode name ;

$(3047-9)$

That good Arcite, of chivalrye flour

Dans The Knightes Tale de G. Chaucer, le motif courtois est principalement polysémique et icônique ' (c'est-à-dire fort de symboles) comme le prouvent les vers 3048 et 3059 .

Rappelons d'abord le contexte du vers 3048. Le syllogisme fondamental : «in his excellence and flour» est d'abord exposé d'une manière rationnelle (voir les trois premiers vers), puis il devient sousjacent et implicite dans l'image florale dans laquelle la jeunesse et la vieillesse sont associées par la mort qui survient tôt (ceci est clairement explicité dans les derniers vers du passage : «in youthe or elles age, / He moot ben deed, the king as shal a page»). En guise d'exemple ou de démonstration, la leçon souligne le parallèle avec le chêne «Lo the ook» (3017) et avec les pierres : «The grete tounes» (3025), tous deux soumis à cette inexorable dégradation. Aussi, nous ne manquerons pas de souligner que la fleur évoque la beauté de la jeunesse : le parallèle entre la beauté du 
corps et celle de la réputation introduit ensuite la beauté cachée de l'âme, de sorte que la mort est embellie par la jeunesse, garante de la pureté. Ainsi, la métaphore florale métamorphose la laideur de la mort à la manière d'une couronne de laurier.

$\mathrm{Au}$ vu de ces remarques, «in his excellence and flour» met l'accent sur la jeunesse et la vigueur du chevalier prêt à se donner la mort pour la femme qu'il aime, et cela par analogie avec les vertus ou les qualités d'une fleur, laquelle afficherait dans ses beaux jours un spectacle de fraîcheur et de robustesse.

Néanmoins, la relation analogique homme / nature, éclairée par la métaphore, crée une distance entre le lecteur et l'appréciation qualitative connotée par l'image de la fleur. Le transfert sémantique se limitant au champ visuel, les sens ne sont pas mis en éveil. Ceci est explicité plus loin, au vers 3059: «That good Arcite, of chyvalrye flour» où le motif de la fleur, connotant en réalité l'isotopie de la chevalerie toute entière, ne renvoie aux qualités intrinsèques du personnage et à ses vertus que par le filtre de la distanciation poétique.

Ceci étant, la métaphore de la «fleur de la chevalerie» renvoie aussi à une image symbolique, proche du blason : on peut y lire et y voir les fleurs de lys que portaient les chevaliers partisans de Richard II (1377-99) lors des tournois et des combats. À cet effet, plutôt qu'une qualité du personnage, ce motif devient la marque d'une convention poétique ${ }^{8}$. La fleur empruntée au registre de la nature devient un ornement littéraire et nous fait entendre la voix du poète courtois. Moins que le récit d'événements, à savoir la mort de l'amoureux, c'est le discours du poète qui est mis en lumière, l'image florale servant ici de relais entre le récit et la mise en discours.

Tomber dans le piège de la catachrèse, c'est-à-dire de la métaphore figée, ne signifie pas pour autant demeurer insensible au monde extérieur, et bien que le contact avec ce dernier reste au stade expérimental, le lecteur moderne ne peut s'empêcher de faire le parallèle entre cette image et celle de St François prêchant aux oiseaux, représentée vers 1296 par le peintre italien Giotto". Dans cette fresque naturaliste avant l'heure, l'ornement végétal des pages enluminées des manuscrits gothiques fait place à un seul arbre, lequel devient métonymie de la forêt toute entière. L'arbre de Giotto est inscrit dans le récit d'événement, alors que les entrelacs gothiques font référence à la page elle-même, à l'ornement et au talent de l'artiste, comme l'illustre, à titre d'exemple, le Trinity Roll de $1517^{10}$ représentant Henry. VIII dans une lettrine, reproduisant la première 
initiale des Plea Rolls, à savoir la lettre $\mathrm{P}$, laquelle sort du cadre et déploie tout le talent et l'ingéniosité du calligraphe gothique à transformer la marge en un ornement, voire une production artistique de l'esprit. Toutefois, et pour revenir ne serait-ce qu'un instant sur la fresque de Giotto, la nature n'en est pas moins omniprésente dans l'entrelacs, le feuillage abritant souvent des oiseaux, semi-figuratifs. Ceux-ci paraissent en effet habiter le feuillage, une partie de leurs ailes disparaissant derrière les rameaux. Quant à l'entrelacs lui-même, il repose sur l'apparition et la disparition des rameaux, suggérant une épaisseur, voire une densité à la toile.

\section{b) La nature comme source de jeu sur les mots}

L'omniprésence de la Nature dans les jeux sur le sens des mots est aussi une caractéristique du motif floral. Chez Chaucer, la tendance à la synesthésie qui se restreint à la projection et à la propagation visuelle du signe poétique et qui opte pour une dialectique hermétique semble pourtant, par le recours aux proverbes, avoir prise sur le monde réel. Le vers 1522 de The Knightes Tale mérite qu'on s'y attarde : «Feeld hath eyen, and the wode hath eres». Il s'agit ici de la vulgarisation d'un proverbe qui, selon Walter W. Skeat, serait la transposition en langue vernaculaire d'un proverbe largement répandu en France et en Angleterre en latin médiéval : «Campus habet lumen, et habet nemus auris acumen»", que l'on peut traduire comme suit : «le champ est pourvu de lumière et le petit bois d'une oreille». Dans le vers de Chaucer, l'équilibre syntaxique, renchéri de part et d'autre de la césure par l'écho de l'auxiliaire «have» («hath»), confère aux prés et aux bois des qualités sensorielles, et non plus seulement des ressemblances. Au lieu d'une comparaison, voire d'une métaphore, il s'agit ici d'une nature anthropomorphique, pourvue des mêmes sensations que le lecteur et le poète, c'est-à-dire dotée d'yeux et d'oreilles. Ainsi, une intimité toute nouvelle se tisse entre l'homme et la nature, favorisant l'humanisation de cette dernière, sorte de brèche dans le discours courtois où dorénavant le seuil est franchi pour jeter un regard nouveau sur ce qui demeurait étranger, distant de l'homme, à savoir la nature elle-même.

\section{2) La Fleur chez les Élisabéthains}

Pendant la Renaissance, et comme l'illustrent les vers de Spenser dont il va être question ainsi que le discours du jardinier dans l'acte III de Richard II, on constate une meilleure intégration du motif floral dans le 
récit d'événements ${ }^{12}$. Cela signifie que la topique du floral a enfin trouvé un socle diégétique qui lui convient pour s'exprimer pleinement. L'enrichissement des récits, preuve d'un renouveau de la pensée à la Renaissance, se manifeste par la circulation des manuscrits, la prolifération des traductions en langue vernaculaire de manuscrits anciens, accordant ainsi au livre un nouveau crédit.

\section{a) Spenser et le carpe diem}

The whiles some one did chaunt this louely lay;

Ah see, who so faire thing doest faine to see, In springing flowre the image of the day; Ah see the Virgin Rose, how sweetly shee Doth first peepe forth with bashfull modestee, That fairer seemés, the lesse ye see her may ; Lo see soone after, how more bold and free Her bared bosome she doth broad display ; Loe see soone after, how she fades, and falles away.

So passeth, in the passing of a day, Of mortall life the leafe, the bud, the flowre, Ne more doth flourish after first decay, That earst was sought to decke both bed and bowre, Of many a Ladie, and many a Paramowre :

Gather therefore the Rose, whilest yet is prime, For soone comes age, that will her pride deflowre : Gather the Rose of loue, whilest yet is time, Whilest louing thou mayst loued be with equall crime.

Chez Spenser, l'extrait du livre II du chant 12 fait l'apologie de la «Virgin Rose», c'est-à-dire de la Vierge Rose ou de la Rose Vierge, persistance de l'image florale dont il vient d'être question. Toutefois le lyrisme s'inspire ici de la tradition du Bel Canto italien, plagiant à quelques inflexions près les octaves 14 et 15 du seizième chant de la Jérusalem Délivrée du Tasse (vers 1565). Or, ce que le modèle italien a apporté, c'est l'épanouissement de la voix et du chant, véhicules du spectacle visuel et des émotions, tandis que chez Chaucer, il s'agit d'un déplacement produisant un être hybride : un chevalier fleur ou une prairie lecteur et poète. Chez le Tasse, le motif floral devient lyrique :

Deh mira - egli canto - spuntar la rosa 
dal verde suo modesta e verginalla,

À la lecture de ces vers, on ne peut que garder à l'esprit la métaphore de Boccace, qui dans son Décaméron, nous parle des «oreilles du cœur»". Le lyrisme de l'extrait cité ci-dessus tient à la forte oralité du message, qui par la voix du discours, à savoir par un procédé appelé en narratologie le débrayage, amorcé par la locution «Deh mira», interpelle directement le lecteur, afin de mieux lui faire partager sa chanson, autrement dit son récit : «egli canto». À ceci, Spenser, dans les deux premiers vers de la strophe 74 ( $\ll$ The whiles some one did chaunt this louely lay; / Ah see, who so faire thing doest to see»), utilise lui aussi la métalepse énonciative, à savoir le débrayage du discours au récit, mais cette fois comme s'il s'agissait d'un ornement poétique. En effet, l'invocation «Ah see», reprise de manière anaphorique et systématique aux vers 4,7 et 8 , sert de refrain dans le chant afin de reconstituer par le pouvoir de ce dernier ( $\ll$ did chaunt this louely lay») une scène visuelle absente. À cet égard, il y a bien synesthésie et transfert d'un registre sensoriel à l'autre.

Toutefois, la thétorique spensérienne conserve les reliquats de la poésie courtoise médiévale. Toujours à la strophe 74 , le regard («Ah see», «Lo see soone after») souligne l'éclat de la figure hybride Femme / Rose, et plus précisément la modestie : «bashfull modestee» (5), et la générosité : «bold and free» (7). Il s'agit ici d'une figure de rhétorique dont on trouve trace dans Le Roman de la Rose de Guillaume de Lorris et Jean de Meun, nommée l'Inamorammento ${ }^{16}$, grâce à laquelle le poète parvient à «toucher le cœur en traversant l'œil». Par ailleurs, la strophe 75 du chant $12 \mathrm{du}$ livre II de The Faerie Queene reprend le thème du carpe diem emprunté à Catulle" : «Gather the Rose, whilest yet is prime» (6), la catachrèse faisant perdre au texte son intensité lyrique et emphatique, ce qui n'est pas le cas des vers de Richard II comme nous allons le voir à présent.

b) Shakespeare et le jardin sur scène

(Queen and Ladies retire.

Gard. Go, bind thou up yon dangling apricocks, Which, like unruly children, make their sire Stoop with oppression of their prodigal weight ; Give some supportance to the bending twigs. Go thou, like an executioner Cut off the heads of too fast growing sprays That look too lofty in our commonwealth : 
All must be even in our government.

You thus employed, I will go root away

The noisome weeds which without profit suck

The soil's fertility from wholesome flowers.

La scène 5 de l'acte III de Richard II utilise la symbolique du floral pour jeter différents regards sur la scène politique, en l'occurrence sur l'imminente déposition de Richard II. L'ouverture de l'allégorie politique par le discours du jardinier, jetant l'anathème sur, à la fois, l'inaptitude du roi à gouverner et sur la mauvaise santé du royaume, présente au spectateur une mise en scène du lieu-commun qui lui confère une étonnante vraisemblance.

Reprenant le schéma analogique médiéval qui pose la Nature comme le prédicat pour exalter les passions humaines, l'adresse du jardinier à son aide-jardinier sous le mode impératif : «Go, bind thou up» (vers 29), repris plus loin par «Go thou» (33), offrent d'une part, une lecture symbolique au spectateur de la scène et, d'autre part, une impression fortement naturaliste rappelant la fresque de Giotto. La musicalité de certains verbes comme «bind thou up», ou encore «Cut off» au vers 34 exposent à la fois le spectacle du jardinier qui somme son assistant d'attacher les jeunes plants de crainte qu'ils n'étouffent les fruits mûrs et ne les entrâ̂nent à leur perte, et celui du travail du jardinier sur scène.

Ce spectacle horticole, lieu-commun hérité de la poésie courtoise, se double d'un autre spectacle, symbolique cette fois-ci, que la rhétorique rend manifeste, à savoir le vent de protestation qui anime les dissidents de l'actuel roi. Dans «bind up», la labiale mime phonétiquement le cercle que forme la bouche en la prononçant, représentant, de manière tactile et visuelle, le spectacle allégorique de la tâche du roi Richard qu'il n'a pas su accomplir. Quant à «Cut off», il signale aussi la séparation que le roi aurait dû s'imposer. Toutefois, on peut aussi comprendre «Cut off» comme un écho à III.1 de la sordide tâche du bourreau qui de son épée ou de sa hache exécute Bushy et Green (III.1), dont on remarquera au passage les métaphores florales. Ainsi, le jardin allégorique offre, tour à tour, un spectacle visuel, sonore et tactile où les sens, motivés par une seule et même topique à l'origine, à savoir celle du floral, parviennent à recréer le climat d'incertitude et de tension politique inaugurant la Guerre des Deux Roses à venir.

$\mathrm{Au}$ vu de ces exemples, la «langue des oiseaux» - c'est-à-dire l'écriture elle-même - mérite d'être reconsidérée de plus près. 


\section{La «langue des oiseaux» démystifiée}

\section{1) L'allégorie figurative chez Chaucer}

À la mutation thématique et historique du floral viennent s'ajouter, et ce dans un registre plus rhétorique, l'émergence de figures nouvelles telles que la synesthésie, lesquelles en osmose avec le récit parviennent, comme nous le verrons, à démystifier le parler courtois, lui donnant ainsi une dimension réaliste. Cette victoire du récit d'événements sur le discours poétique va de pair avec l'essor de l'écriture et du «bien parler» cicéronien propre à la philosophie de la Renaissance, où l'effacement du narrateur est une caractéristique de l'art classique. À titre d'exemple iconographique, la bordure classique, qui perd sa fonction de marge gothique pour devenir un cadre à part entière, ne manque pas d'orienter l'œil du spectateur à l'intérieur même du cadre, là où précisément le message prend toute sa valeur. La disparition progressive de l'ornementation médiévale apparaît, et ce à titre d'illustration, sur la lettrine du Easter Roll de $1584^{19}$, où la bordure même constitue un cadre autonome, distinct de l'image de la reine, et qui de plus est décorée de la manière la plus classique qui soit, à savoir reproduisant des rinceaux de manière géométrique, à la manière des feuilles d'acanthes qui ornent les pilastres de l'époque corinthienne.

Dans la littérature, l'utilisation du floral - et plus précisément le motif de l'arbre - parvient, comme nous allons le voir, et ce en relation avec l'évolution artistique, à se charger de sens jusqu'alors étouffés par l'écriture ornementale. Ainsi l'arbre généalogique devient le témoignage du sentiment de noblesse, comme nous le rappelle Dante dans sa Divine Comédie :

noblesse

a) L'arbre généalogique et l'expression du sentiment de

L'humaine probité ressurgit rarement dans ses branches ; et celui qui la donne le veut ainsi, pour qu'on la lui demande.

Wel can the wyse poete of Florence, . That highte Dant, speken in this sentence ; Lo in swich maner rym is Dantes tale :

"Ful selde up ryseth by his branches smale prowesse of man ; for god, of his goodnesse, 
wol that of him we clayme our gentillesse ;"

$$
(1125-30)^{21}
$$

Le sage poète de Florence,

Qui s'appelait Dante, sait bien exprimer cela.

Ecoutez : voici un vers exemplaire du conte de Dante :

'Tant rares fois remonte vers le haut par les petites branches

Prouesses d'ancesseurs : car Dieu, dans sa bonté,

Veut que ce soit de lui que nous réclamions notre noblesse'.

$(1125-30)^{22}$

La codification de ces vers de Dante que l'on retrouve dans le conte de The Wife of Bath de G. Chaucer cité ci-dessus : «Ful selde up ryseth by his branches smale prowesse of man; for God, of his goodnesse, wol that of Him we clayme our gentillesse», n'offre au lecteur qu'une lecture métaphorique très elliptique et fort éloignée de l'allégorie qu'elle masque, laquelle se devine en filigrane derrière le voile obscur des mots. On distingue à demi l'allégorie de l'Arbre de la Grâce et de la probité introduite par l'allusion à un autre arbre, l'Arbre Dynastique, rendu explicite par la synecdoque «his branches». De plus, la syntaxe elle-même pourrait être glosée comme suit : «la bravoure ne pouvant jaillir de ses branches», où la bravoure, notion chevaleresque, fait écho à l'une des quatre vertus cardinales, à savoir le courage. Quoiqu'il en soit, la figure hybride Homme / Arbre est bel et bien présente, soulignée par la poésie. L'effet d'allitération en $/ \mathrm{r} /$ qui caractérise l'ensemble : «ryseth», «branches» et «prowesse» crée une cohésion sonore, visuelle et sémantique, figurativisant par le jeu musical l'arbre dynastique, caché derrière le rideau métaphorique.

\section{b) L'arbre et l'expression des vices, ancêtre des passions}

\section{«The Tree of Penitence»}

The rote of this tree is Contricion, that hydeth him in the herte of him that is verray repentant, right as the rote of a tree hydeth him in the erthe.

Of the rote of Contricion springeth a stalke, that bereth braunches and leves of Confession, and fruit of Satisfaccion.

For which Crist seith in his gospel : 'dooth digne fruit of Penitence' ; for by this fruit may men 
knowe this tree, and nat by the rote that is hid in the herte of man, ne by the braunches ne by the leves of Confession.

$(\S 6,113-4)$

«The Tree of Pryde»

Of the roote of thise sevene sinnes thanne is Pryde, the general rote of alle harmes; for of this rote springen certein braunches, as Ire, Envye, Accidie or Slewthe, Avarice or Coveitise (to commune understondinge), Glotonye, and Lecherye.

And everich of thise chief sinnes hath hise braunches and his twigges, as shal be declared in hir chapitres folwinge.

$$
(\$ 23,387-8)^{23}
$$

Dans The Persones Tale de G. Chaucer, un pas de plus vers la représentation est franchi, lorsque le motif de l'Arbre, allégorie des Vices et des Vertus, est entendu comme une extension de l'Arbre de Jessé. Si ce dernier était précédemment élidé et faiblement suggéré par la notion de Gentillesse, c'est-à-dire par la Grâce même comme le suggère Dante dans son Convivio ${ }^{24}$, l'Arbre de la Pénitence du Persones Tale, est en revanche décrit et donc exposé au lecteur avec le plus grand soin. Les ingrédients de l'allégorie qui se rapprochent, faut-il le souligner, de l'allégorie personnifiée, sont présents : la relation d'inclusion, amenée par l'auxiliaire être, «is», confère aux racines de cet arbre une qualité humaine : la Pénitence («Contricion») laquelle se loge dans le cœur de l'homme repentant. À ceci il faut ajouter l'apposition : «that hydeth him in the herte of him that is verray repentant». Celle-ci est embrassée, de part et d'autre, par une description de l'arbre allégorique, resserrant l'analogie Homme / Arbre, et personnifiant ce dernier. Le travail sur la syntaxe se double à nouveau d'un jeu sur les sonorités: l'allitération en $/ \mathrm{h} /$ regroupant «hydeth» - qui apparaît sur deux registres, à savoir le floral et l'humain, dénotant l'arbre de la contrition et parallèlement l'âme de l'homme «herte» et «him» - réitérée et donc mis en lumière - permet non seulement de rapprocher l'Arbre de la Pénitence de l'Homme vertueux, c'est-à-dire celui qui fait l'effort de se repentir, mais octroie à la nature des qualités humaines. Par la musicalité du vers, l'Arbre de la Pénitence se 
«herte» et «him» - réitérée et donc mis en lumière - permet non seulement de rapprocher l'Arbre de la Pénitence de l'Homme vertueux, c'est-à-dire celui qui fait l'effort de se repentir, mais octroie à la nature des qualités humaines. Par la musicalité du vers, l'Arbre de la Pénitence se dote d'un cœur, et tout naturellement diffuse par ses branches et ses fruits la confession et la satisfaction.

Quant à l'arbre d'orgueil, «The Tree of Pryde» de The Parsones Tale, si la syntaxe demeure très semblable à celle utilisée plus haut pour décrire l'Arbre de la Pénitence, le choix du lexique, en l'occurrence la juxtaposition des vices, crée un crescendo visuel et sonore chez le lecteur, personnifiant ainsi les vices et portant sur eux un jugement appréciatif et qualitatif. Bien au-delà du cortège des figures allégoriques qui semblent prendre place à la cime des branches de l'Arbre d'Orgueil, la lecture des vices fait naître le spectacle absent des émotions. Le lecteur ne manque pas de les intérioriser et de les assimiler à son comportement. Ceci étant, la dimension sensorielle découlant de la description, demeure chez Chaucer, à l'état de gestation, et ne commencera vraiment à s'épanouir qu'à la Renaissance.

2) Le maniérisme ${ }^{25}$ et la naissance de la nature humaine

Si l'émotion demeure implicite chez Chaucer et n'est exprimée que par la musicalité des vers et la manipulation syntaxique (indissociable des règles du decorum), la poésie et le théâtre élisabéthain parviennent à harmoniser ces paramètres, et surtout à franchir le seuil allégorique; le motif biblique de l'Arbre et des fruits se sécularise, voire se lexicalise, pour exprimer dans les moindres détails réalistes (ou maniéristes) les passions humaines.

\section{a) La représentation des sens chez Spenser}

Yet him in euery part before she knew, How euer list her now her knowledge faine, Sith him whilome in Britaine she did vew, To her reuealed in a mirrhour plaine, Whereof did grow her first engraffed paine ; Whose root and stalke so bitter yet did tast, That but the fruit more sweetnesse did containe, Her wretched dayes in dolour she mote wast, And yield the pray of loue to lothsome death at last. ${ }^{26}$ 
Dans la strophe 17 du chant III de The Faerie Queene l'amour de Britomart pour Arthegall est révélé au lecteur à travers le filtre du topos de l'Arbre des Vices et des Vertus, et ce, contextualisé en un Arbre de la Passion humaine. La rhétorique chemine vers une superposition des champs lexicaux : l'hypallage sur «engraffed paine» (5) met l'accent sur le floral et l'humain, sans pouvoir les dissocier. À la douleur physique, rendue explicite par le syntagme «paine», vient se greffer un cortège d'émotions. Les qualificatifs, suivants : «bitter» (6) et «sweetnesse» (7), introduits dans la strophe par l'hypallage, deviennent des syllepses ${ }^{27}$. Au sens littéral, ces adjectifs donnent une valeur gustative à la plante, et métaphoriquement, se resémantisent, transposant l'impression gustative de ce qui serait bon ou mauvais au palais, en émotions vécues, soit précisément l'amertume mêlée au plaisir d'être amoureux.

\section{b) L'expression de la douleur chez Shakespeare}

Enter Mortimer, brought in a chair, and Jailers.

Mor. Kind keepers of my weak decaying age,

Let dying Mortimer here rèst himself.

Even like a man new haled from the rack,

So fare my limbs with long imprisonment ;

And these grey locks, the pursuivants of death,

Nestor-like agèd in an age of care,

Argue the end of Edmund Mortimer.

These eyes, like lamps whose wasting oil is spent,

Wax dim, as drawing to their exigent ;

Weak shoulders, overborne with burdening grief,

And pithless arms, like to a withered vine

That droops his sapless branches to the ground.

Yet are these feet (whose strengthless stay is numb,

Unable to support this lump of clay)

Swift-wingèd with desire to get a grave,

As witting I no other comfort have.

Quant au spectacle de la douleur au théâtre, la scène du martyre de Mortimer exprime la douleur physique qui précède la mort. Celle-ci se manifeste par une impression tactile de mollesse et d'accablement s'abattant sur tout le corps. Le spectacle du corps vampirisé par la mort s'emparant de sa vigueur, voire de sa «sève»: «like to a withered vine / That droops his sapless branches to the ground» (11-2), transforme cette masse auparavant vigoureuse en un poids mort. «Overborne» et 
«burdening» (vers 10) sont par l'allitération en /b/ homophoniques et homonymiques, soulignant ainsi la tautologie. Leur utilisation révèle une surenchère lexicale qui finit par rendre la scène hyperbolique, en amplifiant la sensation de lourdeur et en insistant sur son aspect inhabituel, voire déjà mortel. Les épithètes «Weak» et «pithless» (vers 10 et 11), qui font écho à «withered» et «sapless» (vers 11 et 12), évoquent le spectacle de la vigne flétrie laissant tomber à terre ses branches desséchées, sorte d'écho aux livres d'emblèmes. En d'autres termes, l'utilisation du comparatif «like to» très voisin de «as» en anglais, sert plus à rendre explicite un état de l'être, en l'occurrence sa «douleur», qu'à suggérer une analogie entre l'homme et le floral.

Avec la mise en scène théâtrale, l'avènement d'une représentation directe, émancipée du discours «florissant» du narrateur médiéval, permet l'utilisation par le personnage-locuteur des images jusque-là réservées au seul narrateur. L'exemple de Mortimer montre autant que celui du jardin, où même celui du monologue du roi Richard II dans la scène 5 de l'Acte V, le mécanisme de la synesthésie comme figure de l'expression du sentiment. Avec le théâtre, nous assistons à une prise en charge par le personnage du motif floral pour exprimer ses propres sentiments. Il y a donc un effet de sens qui suggère la synesthésie, à savoir que la fleur évoque à la fois la vue, l'odorat et le toucher, permettant ainsi de créer des effets sonores, comme l'assonance et l'allitération dans le discours du poète. Tout cela considéré et prenant pour paradigme la littérature courtoise et lyrique du Moyen-Âge qui voit s'épanouir l'instance narrative grâce aux Novella et aux Romances, il serait donc plus cohérent d'envisager la littérature médiévale, en reprenant la métaphore du floral, comme s'il s'agissait d'un bourgeonnement d'émotions et celle de la Renaissance comme d'une floraison.

Nathalie FAURÉ Université Toulouse-le-Mirail 
IABLEAUX

\section{SYNESTHÉSIE ET ÉVOLUTION DU LANGAGE POÉTIQUE}

L'Écriture au Mioyen Age

Axe Paradigmatique (vertical)

Énde des Figures de Rhêtorique:

*On souligne la pauvreté des

tropes qui sont concentres autour

des metaphores d'usage

(ou catachrèses), de l'Allégorie

et de la Prosopopée.

*Ces figures sont autonomes,

détachées de la storia, et n'ont

souvent qu une valeur décorative pour le lecteur.

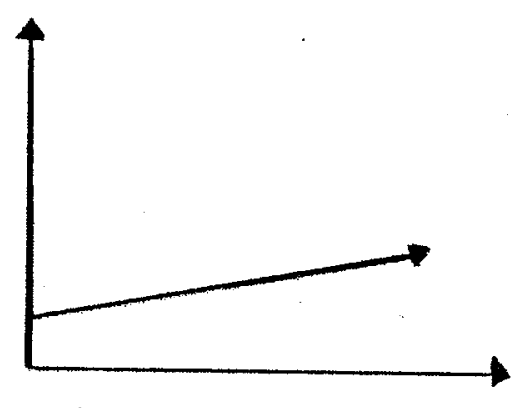

Axe Syntagmatique (horizontal)

Niveau syrraxique :

*Variable et Evolutir depuis

les XIIle \& XIVe siècles.

L'Écriture à la Renaissance

Axe Paradigmatique (vertical)

Étude des Figures de Rhétorique: *Elles s'étoffent, et en relation avec la diégèse (la storia),

elles perdent leur valeur décorative pour se charger de valeurs émotionnelles et poétiques

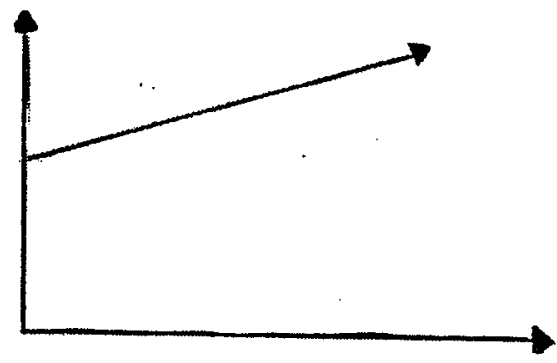

Axe Syntagmatique (horizontal)

Niveau symtaxique:

*Prolifération des récits.

et enrichissement du savoir. 


\section{N O T E S}

'L'Âge d'Or de la Scolastique est une période qui s'étend environ de 1228, début de l'enseignement d'Albert le Grand à Cologne, jusque vers 1350, date de la mort de Guillaume d'Ockham : Étienne Gilson, La Philosophie au Moyen-Âge, Des origines patristiques à la fin du XVIe siècle, coll. Rivages, Paris, Payot (1986), 1999, p. 591.

La théologie spéculative, relative au XIIe siècle fut ensuite remplacée par le Nominalisme, à savoir par la victoire de la logique, de la raison, de la grammaire et plus généralement de la philosophie d'Aristote jusqu'alors ressentie comme une menace pour la foi chrétienne. N.S. Thompson, Chaucer, Boccaccio, and the Debate of Love, A Comparative Study of the Decameron and The Canterbury Tales, Oxford, Clarendon Press, 1996, p. 47 : «In this respect both authors reflect the Nominalist climate of the fourteenth century, which saw an end to the dominance of speculative theology and philosophy: interest turned to epistemology in a new anthropocentric conception of the universe».

'Métalepse reste à être nuancé. En effet, nous n'entendrons par métalepse non pas la figure de rhétorique, mais plutôt l'effet de sens énonciatif produit dans le texte littéraire. Voir Gérard Genette, Figures III, Paris, Seuil, 1972, p. 243 : «Le passage d'un niveau narratif à l'autre ne peut en principe être assuré que par la narration, acte qui consiste précisément à introduire dans une situation, par le moyen d'un discours, la connaissance d'une autre situation».

"Meyer Howard Abrams, A Glossary of Literary Terms, 6th ed., Fortworth, Harcourt Brace Jovanovich, 1993, p. 210. Je dois cette définition à Ingrid Delpech pour sa communication de juin 2000 lors de la journée de l'ASF tenue à l'Université de Toulouse-le-Mirail.

'Gaston Bachelard, L'Intuition de l'instant, coll. Stock, Paris, Denoël, 1992 (1931), p. 106-7.

Geoffrey Chaucer, The Complete Works, Oxford, Clarendon Press, Rev. Walter W. Skeat ed., 1894, «The Knightes Tale», p. 438 \& 456.

'En référence aux codes de l'analyse picturale, nous établissons une distinction graphique et sémantique entre iconique et icônique. Selon Daniel Arasse, Le Détail : Pour une histoire rapprochée de la Peinture, coll. Champs, Paris, Flammarion, 1996, p. 268 : «le premier détail imite un objet ou une partie d'objet; il vise à reproduire ce qu'il représente et à le donner à voir dans sa ressemblance, poussée éventuellement jusqu'au moindre détail. Il fait image ; pour cela on l'appellera détail 'iconique'». Nous opposerons donc l'icone à l'icône religieuse, byzantine en particulier. Voir Martine Joly, L'Image et les Signes, Approche Sémiologique de l'image fixe, Paris, Nathan, 1994, p. 47 : «Dans la 
religion chrétienne et plus particulièrement byzantine, on appelle 'icônes' les images religieuses, indépendamment de leur technique. Images peintes du Christ, de la Vierge Marie, des anges et des saints, elles font l'objet d'un culte privé et aussi liturgique dans les Églises orientales. Elles sont peintes selon les règles très strictes et ne prétendent pas à une quelconque ressemblance».

Cf. Henry VII, Trinity Roll, 1517 (K.B. 27 / 1024), Title Membrane, 76.3 x $22 \mathrm{cms}$ et détail à droite de la membrane «Henry VIII with Heraldic Emblems» (actual size), dans Erna Auerbach, Tudor Artists, A Study of Painters in the Royal Service of Portraiture on Illuminated Documents from the Accession of Henry VIII to the death of Elizabeth I, London, The Athlone Press, planches 52 a et 8.

Cf. Giotto, Saint François prêchant aux oiseaux, 1296-1297, Italie, Fresque de la basilique de Saint-François à Assise, dans Eugenio Battisti, Giotto, Genève, Albert Skira éd., 1990 (1960), p. 21.

${ }^{10}$ Voir note 8.

"Walter W. Skeat, The Knight's Tale or Palamon and Arcite by Geoffrey Chaucer done into Modern English, London, The 'De la More Press', Alexander Moring ed., 1904, p. 100.

${ }_{13}^{12}$ Je vous renvoie aux tableaux en fin d'article.

Edmund Spenser, The Faerie Queene, Harmondsworth, Penguin Books, Thomas P. Roche ed., 1978, p. 379, Book II (1590), canto 12, strophes 74-5.

${ }^{14}$ Torquato Tasso, Gerusalemme Liberata (1564-77), pref. e note di Lanfranco Caretti, coll. Nuova Universale Einaudi, Torino, G. Einaudi, 1971, p. 478, Canto Sedicesimo, ottavas 14-5 :

Deh mira - egli canto - spuntar la rosa

dal verde suo modesta e verginella, che mezzo aperta ancora e mezzo ascosa, quanto si mostra men, tanto è piu bella.

Ecco poi nudo il sen già baldanzosa dispiega ; ecco poi langue e non par quella, quella non par che desiata inanti fu da mille donzelle e mille amanti.

Cosi trapassa al trapassar d'un giorno de la vita mortale il fiore e 'l verde ; né perché faccia indietro april ritorno, si rinfiora ella mai, né si rinverde. Logliam la rosa in su'l mattino adorno di questo di, che tosto il seren perde ; cogliam d'amor la rosa : amiamo or quando esser si puote riamato amando. 
Et pour la traduction des deux premiers vers de l'octave 14 : Le Tasse, $L a$ Jérusalem délivrée, coll. Les Introuvables, Var, Éditions d'aujourd'hui, 1976, p. 276 , chant 16 , octave 14 :

Vois, chantait-il, vois cette rose sortir de son bouton, Modeste et vierge [...]

${ }^{15}$ Boccace, Décaméron, trad. Christian Bec, Paris, Le Livre de Poche, 1994, p. 786, Dixième Journée, 5e nouvelle : «Les paroles que reçoivent les oreilles du cœur ont beaucoup plus de force que l'on ne croit généralement et presque tout devient possible aux amants».

${ }^{16}$ Guillaume de Lorris \& Jean de Meun, Le Roman de la Rose, trad. Armand Strubel, coll. Lettres Gothiques, dir. Michel Zink, Paris, Le livre de Poche, 1992, p. 127 , vers $1738-42$ :

Il trait a moi sanz menacier

La flecheou n'ot fer ne acier

Si que par l'œil ou cuer m'entra

La saiete qui n'en istra james.

Ce même topos réapparaît, notons-le au passage, dans The Knightes Tale de G. Chaucer (1. 1077-9), lorsque Palamon aperçoit pour la première fois Émilie de la tour de sa prison :

He caste his eye upon Emelya,

And there-with-al he bleynte, and cryde 'a !'

As though he stongen were un-to the herte.

${ }^{17}$ Torquato Tasso, Gerusalemme Liberata, Canto Sedicesimo, ottava 15 : «logliam la rosa in su'l mattino adorno / di questo di, che tosto il seren perde». L'adage se retrouve dans la poésie de Catulle, Carmina, éd. H. Bardon, coll. Latomus, Bruxelles, Revue d'Études Latines, vol. 112, 1970, p. 122, épithalame 62, 1. 39-47:

Comme naît une fleur au secret des jardins enclos, ignorée du bétail, sans charrue qui l'arrache; elle est caressée des brises, fortifiée du soleil, et pousse à la pluie : beaucoup de garçons l'ont désirée, beaucoup de filles : mais quand elle s'est fanée, cueillie d'un ongle mince, point de garçons qui la désirent, point de filles. Ainsi la vierge : tant qu'elle se garde intacte, tant elle est chère aux siens ; quand de son corps violé elle perd la fleur de pureté, ni les garçons ne lui gardent leur tendresse, ni les filles leur affection.

${ }^{18}$ William Shakespeare, The Complete Works, London, The Penguin Press, Allen Lane ed., 1969, p. 656, «The Tragedy of King Richard the Second», III.4.2939. 
19

Cf. Elizabeth I, The Easter Roll, 1584, (K.B. 27 / 1289), size 20.1X 15.3 cms, dans Erna Auerbach, Tudor Artists, planche 44.

Alighieri Dante, La Divine Comédie, Le Purgatoire (Purgatorio), trad. Jacqueline Risset, coll. Bilingue, Paris, Garnier - Flammarion, 1992, p. 75, Chant VII, 1. 121-3.

${ }^{21}$ Geoffrey Chaucer, The Complete Works, op. cit., «The Tale of the Wyf of Bath», p. 579.

${ }^{22}$ Geoffrey Chaucer, Les contes de Cantorbéry, trad. Juliette de Caluwé-Dor, Louvain, Peeters, 1986, p. 189 \& 191, «Ici, la femme de Bath finit son Prologue et commence son conte».

${ }^{23}$ Geoffrey Chaucer, The Complete Works, op. cit., «The Persones Tale», p. $676 \& 687$.

${ }^{24}$ Alighieri Dante, Le Banquet (Il Convivio), trad. Philippe Guiberteau, Paris, les Belles Lettres, 1968, p. 314, Quatrième Traité, chap. V.6 :

C'est pour cela qu'il est écrit dans Isaïe : «Un rameau naîtra de la souche de Jessé, et de sa souche une fleur montera».

${ }^{25}$ Jean-Pierre Maquerlot, Shakespeare and the Mannerist Tradition. A Reading of Five Problem Plays, Cambridge, Cambridge University Press, 1995, p. 15 : «In the sixteenth Century, artists were less concerned with exploring new ways of relating to nature - this had been the main preoccupation of the Quattrocento - they were more intent on stating their right to go their own way in face of the relative dogmatism of the High Renaissance. [...] Hence the everincreasing importance of self-expression; hence the ever-increasing need to possess a style. [...] It is significant that all the theorists of this period looked upon the study of the masters and nature merely as a means of strengthening individual giftedness and allowing artists to demonstrate their personal talents». Et aussi p. 19 : «The truth is that behind analogy by withdrawal there lies concealed the old separation between content and form, essence and existence, between a part that is lofty, abstract and meaningful and a part that is inferior, material and contingent, seen as the mere embodiment in various modes, of the Idea behind the artist's work».

${ }^{26}$ Edmund Spenser, The Faerie Queene, op. cit., Book III (1590), canto 2, strophe 17, p. 406.

George Puttenham, The Arte of English Poesie (1589), fac simile, London, Scolar Press, Menston ed., 1970, p. 137 : «[...] by the figure sillepsis, whom for that respect we call [double supplie] conceiuing, and as it were, comprehending under one, a supplie of two natures». Pour une définition plus récente, voir Bernard Dupriez, Gradus, les procédés littéraires, coll. 10/18, Paris, Union Générale d'Éditions, 1984, p. 434 : «Syllepse de sens : figure par laquelle un mot est employé à la fois au propre et au figuré». 
${ }^{28}$ William Shakespeare, The Complete Works, p. 453, «The First Part of King Henry the Sixth», II.5.1-16. 